\title{
Time Constants of the Transition between Onset and Decay Reynolds Numbers for the Appearance of Taylor-Couette Flow
}

\author{
Takashi Watanabe \\ Graduate School of Information Science, Nagoya University, Nagoya, Japan \\ Email: takashi@is.nagoya-u.ac.jp
}

Received January 4, 2012; revised February 1, 2012; accepted February 20, 2012

\begin{abstract}
We investigate the onset and the decay of Taylor-Couette flow in finite cylinders, and we report the estimated time scales in the azimuthal section of the flow state transition between the super-critical state and the sub-critical state by fitting the numerical result to the solution of the Stuart-Landau equation. The inner cylinder rotates, and the outer cylinder and both end walls of the cylinders are stationary. Near the end walls of the cylinders, the value of the time scale is small. In the inner region, the radial velocity component has a large time scale near the center of the vortices, while the axial velocity component has a large time scale between the vortices.
\end{abstract}

Keywords: Taylor-Couette Flow; Onset/Decay Flow; Time Scale; Stuart-Landau Equation

\section{Introduction}

Since the pioneering work of G. I. Taylor, Taylor-Couette flow has been investigated using theoretical, experimental and numerical approaches [1]. In this paper, we investigate the flows between the coaxial rotating inner cylinder and the stationary outer cylinder, and the lengths of the cylinders are finite. The geometrical parameters that determine this flow are the aspect ratio and the radius ratio of the two cylinders and the parameter governing the flow is the Reynolds number defined by the circumferential velocity of the inner cylinder and the gap width of the two cylinders.

Benjamin [2,3] showed, when the lengths of the cylinders are finite, that the bifurcation from Couette flow to Taylor vortex flow is imperfect. Since this study, many flow modes have been discovered, and bifurcation diagrams in the space spanned in the Reynolds number and the aspect ratio have been evaluated $[4,5]$.

Theoretical work has determined the critical Reynolds number for the appearance of Taylor vortex flow from Couette flow [6]. The theoretical value is found only in the flow between infinite cylinders. Recently, the onset and decay of the flow in the range of the Reynolds number including the critical value were investigated by Abshagen et al. [7]. In their numerical calculation, the basic flow is that between the infinite cylinders, and the effect of the end walls of the cylinder was considered by intro- ducing the homotopy parameter. This means that the exact effect of the solid stationary end wall is not estimated. Manneville and Czarny [8] fully considered the end walls and examined their effect. They found that, while the decaying flow follows Landau-type development, the onset flow departs from Landau-type development as the aspect ratio increases. However, both Abshagen et al. and Manneville et al. investigated the timedependent flow at one point, or only in the one-dimensional region whose axis is parallel to the cylinder axes. Therefore, the flow development in the entire azimuthal region is not clear.

In this study, we investigate the onset flow and decay flow in the entire azimuthal region. It is currently difficult to correctly measure the velocity components in the entire region experimentally. Therefore, a numerical approach is adopted here. That is, we assume that the flow state transition caused by the change of the rotating speed of the inner cylinder follows the Stuart-Landau equation, and we determine the initial amplitude, final amplitude and time scale by fitting the numerical solutions to the exact solution. This method is helpful in visualizing the flow conditions of this type of flow. In this paper, we show in particular the result of the time scales at a radius ratio of 0.667 .

Section 3 discusses the formulation and fitting method used, and Section 4 presents the results obtained in this study. Finally, some conclusions are given in Secion 5. 


\section{Formulation}

\subsection{Flow Filed and Governing Equation}

The flow field consists of two coaxial cylinders. Figure 1 shows the diagrammatic figure of the flow field. The length of the cylinders is finite and is denoted by $L$. The end walls of the cylinders and the outer cylinder are fixed and stationary, while the inner cylinder rotates. The radii of the inner and outer cylinders are $R \mathrm{i}$ and $R o$, respectively. The radius ratio $\eta$ is given as $R \mathrm{i} / R \mathrm{Ro}$, and the aspect ratio $\Gamma$ is defined as $L /(R o-R \mathrm{i})$.

The origin of the cylindrical coordinate system $(r, \theta, z)$ is at the center of one end wall. In the numerical formulation, the reference length is the gap width of the cylinder $(R o-R \mathrm{i})$, and the reference velocity is the circumferencial velocity of the inner cylinder, and all of the physical values are made dimensionless by these reference values. Let $u, v$ and $w$ be the velocity components in the radial, azimuthal and axial direction, respectively, and their vector form is represented by $\boldsymbol{u}$. Then, the governing equations are given as follows:

$$
\begin{aligned}
\nabla \cdot \boldsymbol{u} & =0 \\
\frac{\partial \boldsymbol{u}}{\partial t}+\nabla\left(\boldsymbol{u \boldsymbol { u } ^ { T }}\right) & =-\nabla p+\frac{1}{\mathrm{Re}} \Delta \boldsymbol{u}
\end{aligned}
$$

Here, $t$ is time, $p$ is pressure, and Re is the Reynolds number based on the gap width of the cylinders and the circumferential velocity of the inner cylinder.

In the numerical calculation, a staggered grid is used. This grid is useful in avoiding the singularity at the corner between the stationary wall and the moving wall. The convection terms are discretized by the QUICK method, and the central difference method is used for other spatial derivatives. The time integral is performed according to the fractional step method.

The number of grid points in the radial direction is 81 . The number of grid points in the axial direction is 81 for unity aspect ratio, and the number for other aspect ratios is determined by the proportionality to the aspect ratio. Sufficient accuracy has been confirmed using these grid points. The boundary condition at the solid wall is the no-slip condition on the entire solid wall. In the initial condition, the flow is at rest in the entire region.

\subsection{Fitting for the Solution of the Stuart-Landau Equation}

The Stuart-Landau Equation [7].

$$
\tau_{0} \frac{\mathrm{d} A}{\mathrm{~d} t}=\varepsilon A-\frac{A^{3}}{A_{0}^{3}}
$$

is the equation that governs the amplitude near the critical point. Here, $\tau_{0}$ is the time scaling factor. The critical Reynolds number is denoted by $\operatorname{Re} 0$, and $\varepsilon$ is represented

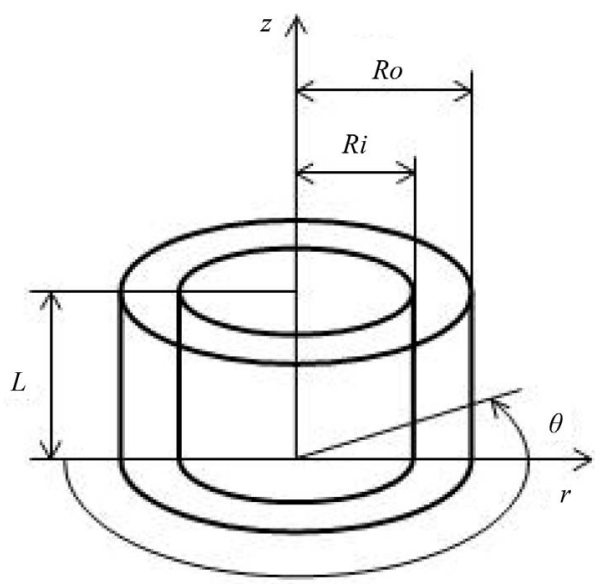

Figure 1. Test flow configuration and coordinate system.

by $(\operatorname{Re}-\mathrm{Re} 0) / \mathrm{Re} 0$. In the present study, the critical Reynolds number is given as the Reynolds number beyond which Couette flow becomes Taylor vortex flow between the infinite cylinders. The solution of this equation is given by

$$
A(t)=\frac{A_{f} \mathrm{e}^{t / T}}{\sqrt{\mathrm{e}^{2 t / T}+\left(A_{f} / A_{i}\right)^{2}-1}}
$$

where $A_{i}$ is the initial amplitude, $A_{f}=A_{0} \sqrt{\varepsilon}$ is the amplitude in the final state, and $T$ is the time scale. This solution represents the initial exponential growth and then the slowing down effect that makes the amplitude remains at a plateau.

From the numerical results, the least square method is used to determine $A_{i}, A_{f}$ and $T$. Several formulations of the least square method are considered. The method that makes the derivations of the accumulation of the square errors with respect to $A_{i}, A_{f}$ and $T$ zeros indicates the most stable solution process. In our calculation, we used this method.

In the case of radius ratio 0.667 , the critical Reynolds number in the infinite length cylinders is 76.5 [6].

We assume that the sub-critical flow appears at $\mathrm{Re}=$ 75.5 and the super-critical flow emerges at $\mathrm{Re}=77.5$. Once the well-developed sub-critical flow and supercritical flow are established, the Reynolds number is suddenly changed to initiate the super-critical flow or the sub-critical flow, respectively. Then, the transition process between the sub-critical state and the super-critical state is estimated.

\section{Results and Discussion}

\subsection{Fully Developed Flow at Sub-Critical and Super-Critical Reynolds Numbers}

Flows in the azimuthal section at the sub-critical Reynolds number 75.5 and the super-critical Reynolds num- 
ber 77.5 are shown in Figure 2. In these panels, vectors are two-dimensional vectors consisting of radial velocity component $u$ and axial velocity component $w$, and the color contour represents the energy of the velocity given by $u^{2}+w^{2}$. The left side is the rotating cylinder and the right side is the stationary outer cylinder.

When the aspect ratio is 4.0 , vortices are found even at $\operatorname{Re}=75.5$. This occurs because the effect of the Ekman pumping driven by the Ekman vortices appears on the stationary end walls, and the induced interior vortices appear. At $\operatorname{Re}=77.5$, interior vortices appear more clearly and we can count four vortices in total.

When the aspect ratio is 8.0, interior vortices do not appear, except the two vortices near the end walls, in the sub-critical state. However, the super-critical state includes interior vortices, and eight vortices appear.

In the flow for the aspect ratio 12.0, the sub-critical state exhibits vortices near the end wall, while no clear vortex appears in the interior section. The super-critical state exhibits twelve vortices in the entire section.

\subsection{Onset and Decay of Flows between Sub-Critical and Super-Critical States}

In Figure 3, the variations of the radial velocity component in the middle region between cylinders are shown as the profiles depending on the axial coordinate $z$. In this case, time is measured from the point at which the Reynolds number is changed. The panels include the flow onset process from the sub-critical state to the supercritical state and the decay process from the super-critical state to the sub-critical state. In the onset process, the well developed flow at $\mathrm{Re}=75.5$ is suddenly subjected to the flow field with the inner cylinder rotating at the Re $=77.5$. Inversely, in the decay process, the Reynolds number 77.5 is abruptly changed to 75.5 . Then, the restabilization is examined. In each panel, profiles are represented by black, blue and red lines as time progresses. For example, the panels at $\Gamma=8.0$ show that the profiles at times 0, 50 and 200 are represented by black, blue and red lines, respectively.

When the aspect ratio is 8.0, the variation of the velocity component near the interior region of the cylinder emerges, though this is not clear in Figure 2. At this aspect ratio, the development of flow almost terminates at $t$ $=200$. In the profile of the onset process, the values near the end walls of the cylinders $(z=0.0,8.0)$ change sharply by $t=50$ and then reach almost their final values at $t=200$, while this does not occur in the profile of the interior regions. Similarly, the values at $t=50$ near the end walls in the decay process change to the final values at $t=200$. This means that the time scales for the onset and decay processes are different at the end wall region and at the interior region. We further discuss this difference in the next section.

The profile at $\Gamma=12.0$ in the sub-critical state shows the variation of the velocity component, even though no
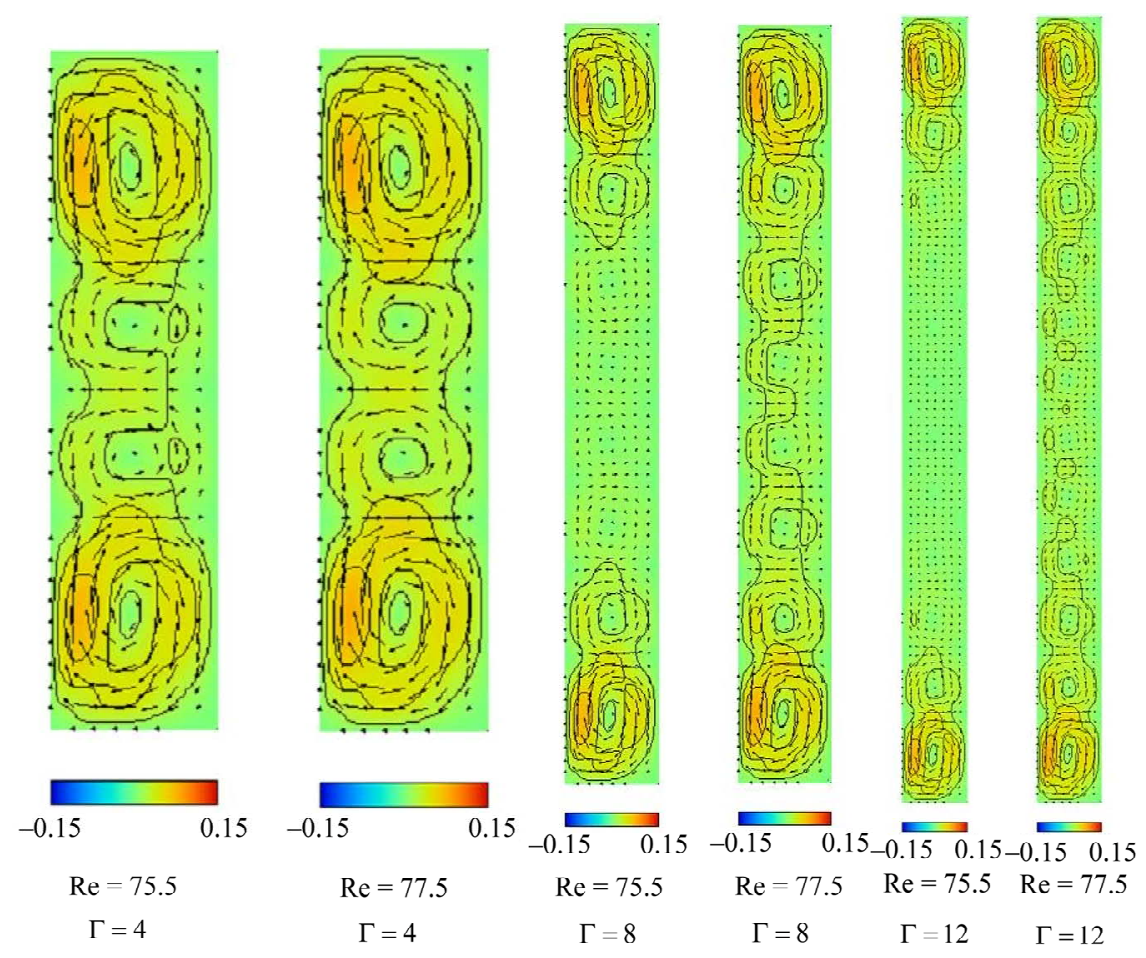

Figure 2. Velocity vector and contour of the kinetic energy in the azimuthal section at the sub-critical Reynolds number 75.5 and the super-critical Reynolds number 77.5 at aspect ratio 4.0, 8.0 and 12.0. 


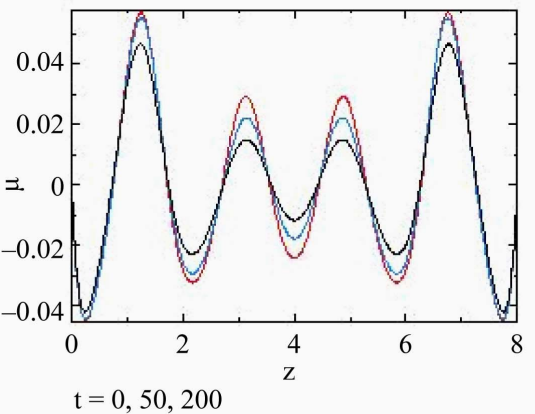

$\Gamma=8$, on set

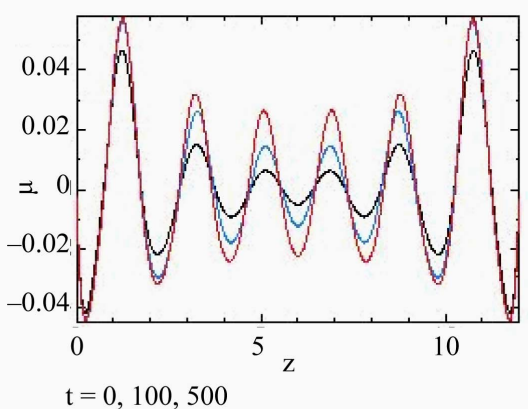

$\Gamma=12$, on set

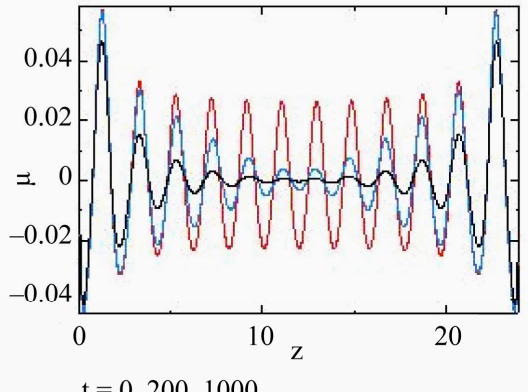

$\Gamma=24$, on set

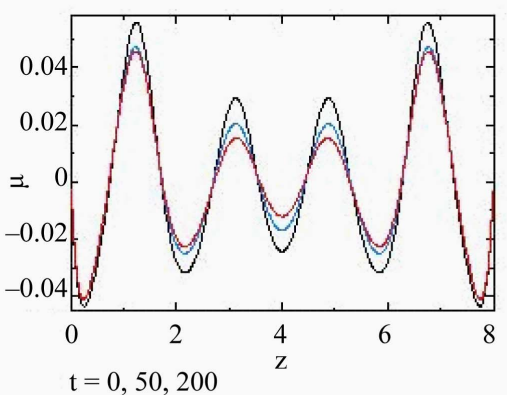

$\Gamma=8$, decay

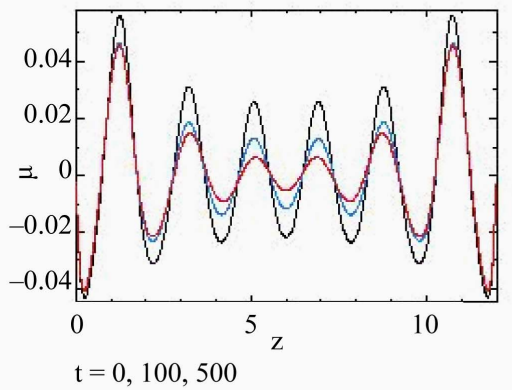

$\Gamma=12$, decay

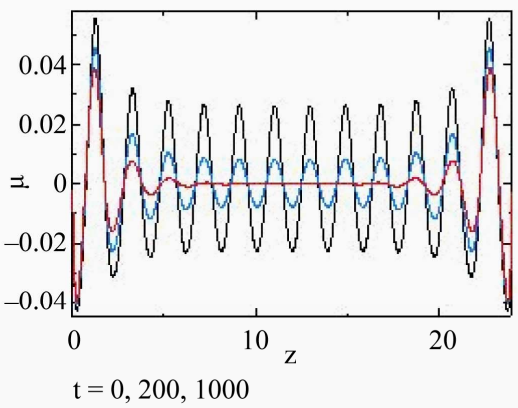

$\Gamma=24$, decay

Figure 3. Onset and decay of flow from the sub-critical state and the super-critical state, respectively. The profiles at the three time points are shown. As time progresses, the profiles are denoted by black, blue and red lines.

obvious vortex is found in the interior region, as shown in Figure 2. The effect of the change in the Reynolds number first appears near the end walls of the cylinders, and then the effect propagates into the interior region of the flow field.

When the aspect ratio is 24.0, the value of the radial velocity component in the sub-critical state remains small at the inner region in the axial direction. On the other hand, the effect of the Ekman pumping appears near the end walls of the cylinder. As the Reynolds number increases, the profile at $t=200$ shows that the value becomes large from the end wall region to the inner region.
At $t=1000$, the profile almost attains its final value. In the decay process, the value in the interior region begins to become small. In the final state at $t=1000$, the value of the profile is almost zero, except in the region that is affected by the Ekman layer.

\subsection{Variation of the Time Scale for Onset and Decay in the Azimuthal Section}

The transient process of the flow is assumed to follow the Stuart-Landau equation, and the time scales at each point in the azimuthal section are estimated. 
The contours of the time scale of the radial velocity component at $\Gamma=8.0,12.0$ and 24.0 are shown in Figure 4. Please note the maximum value of the color bar of the contour.

At aspect ratio 8.0, the time scale remains small near the end walls of the cylinders: that is, the upper and lower ends in the figure. The time scale is relatively large at the center of vortices (please compare the positions at large time scales with the positions of the center of the velocity vector in Figure 2). This means that as the Reynolds number changes, it takes a longer time for the radial velocity component to reach the plateau near the vortex center where the radial velocity component is small. From the legend of the color contour, it can be said that the decay time is at least three times larger than the onset time. This means that the onset and decay in the range of the Reynolds number that includes the critical value are not symmetric with respect to time.

The above results can also be found for the flow at $\Gamma=$ 12.0 and 24.0. First, the time scale is large near the end walls of the cylinders, regardless of the aspect ratio. Second, the time scales near the center of the vortices are large compared with those of the other parts of the flow region, such as areas near the boundaries of vortices.

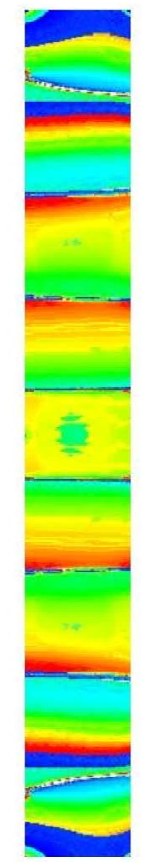

$T \max =100$

onset

$\Gamma=8$
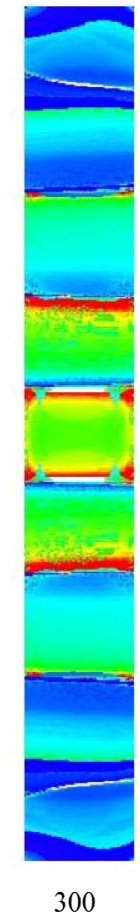

decay

$\Gamma=8$

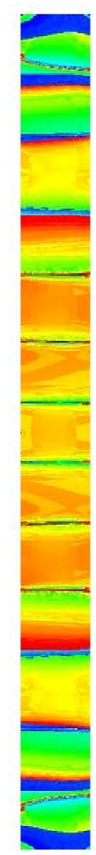

100

onset

$\Gamma=12$
Third, the time scale of the decay is larger than that of the onset.

The contours of the time scale of the axial velocity component are shown in Figure 5. When the flow is onset, the time scale is almost constant except near the end walls of the cylinders, where the value is small. In the decay process, the larger value appears at the inner region of the flow field. The large value appears near the boundaries of the vortices. This is different from the time scale profile of the radial velocity component which has large near the center of vortices.

In the fitting method, we based our approach on the solution given by Equation (4). In the calculation of the real numbers, this equation assumes that $\varepsilon$ is positive. This means that the calculation using only real numbers may not be applicable when $\varepsilon$ is negative and the flow is decay. On the other hand, our fitting method with real number calculations exhibits well-converged result, even when the flow is decay. Abshagen et al. [7] also used Equation (4) as a model of the onset and decay flows and showed good agreement with an experimental result. Furthermore, no $\varepsilon$ explicitly appears in Equation (4). Therefore, at the first glance, Equation (4) is applicable only the onset process, we can estimate the decay as well

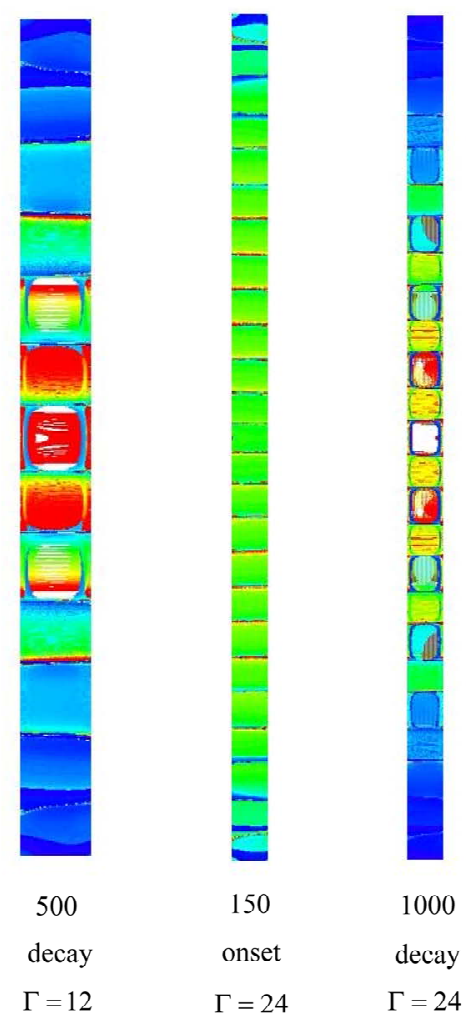

Blue represents the value of 0 and red shows the value of $T$ max

Figure 4. Contour of the time constant of the radial velocity component, estimated from the solution of the Stuart-Landau equation. In the color map , blue represents the value of 0.0 and red shows the value of $T$ max. 


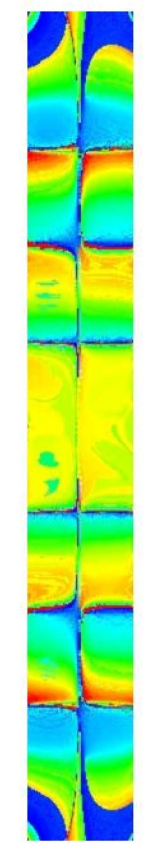

$T \max =100$

onset

$\Gamma=8$

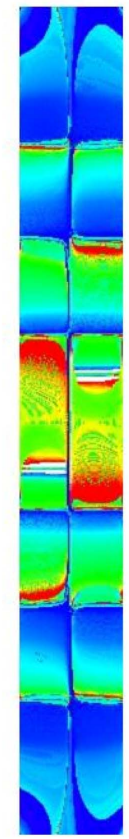

300

decay

$\Gamma=8$

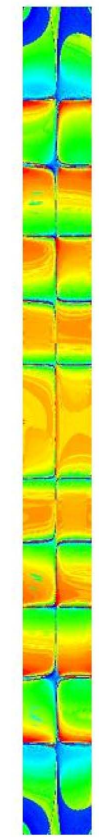

100

onset

$\Gamma=12$

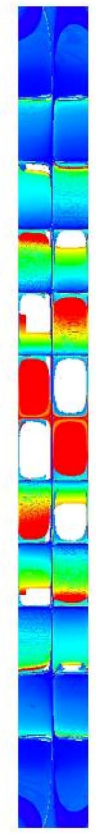

500

decay

$\Gamma=12$
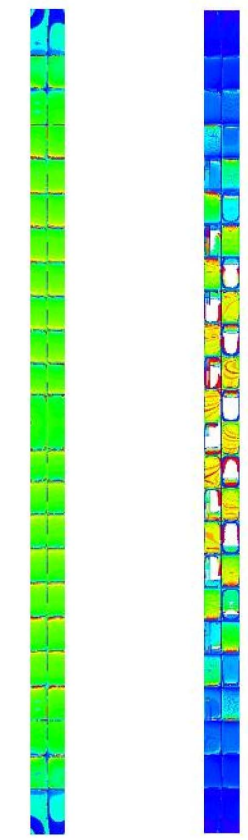

150
onset

1000

decay

$\Gamma=24$

Blue represents the value of 0 and red shows the value of $T \max$

Figure 5. Contour of the time constant of the axial velocity component, estimated from the solution of the Stuart-Landau equation. In the color map blue represents the value of 0.0 and red shows the value of $T$ max.

as onset by using the solution of Equation (3). Further discussion is required on this point.

\section{Conclusions}

In Taylor-Couette flow between a rotating inner cylinder and a stationary outer cylinder, the onset and decay processes found by the change of the Reynolds number in the range including the critical value for the infinite cylinders are examined numerically. In addition, the time scales of the onset and decay processes of the flow are estimated in the entire region of the azimuthal flow section.

When the aspect ratio is small, the Ekman vortices formed on the end walls of the cylinder dominate the entire flow. At the higher aspect ratio, the interior region is independent from the effect of the end wall exists.

For the radial velocity component during the onset and decay processes, the effect of the change in the Reynolds number appears first near the end walls of the cylinder and the effect gradually propagates into the interior region.

The numerical result is fitted to the solution of the Stuart-Landau equation, and the time scales of the onset and decay of the radial and axial velocity components are estimated. The radial velocity component has a small time scale that appears near the end walls, and it has a large time scale that emerges near the center of the vortices. The axial velocity component has a large time scale near the boundary of the vortices at the interior region of the flow section. The time scale of the decay process is larger than that of the onset process, regardless of the radial and axial velocity components.

\section{REFERENCES}

[1] R. Tagg, “The Taylor Couette Problem” Nonlinear Science Today, Vol. 4, 1994, pp. 1-25.

[2] T. B. Benjamin, "Bifurcation Phenomena in Steady Flows of a Viscous Fluid I, Theory", Proceedings of the Royal Society A, Vol. 79, No. 1696, 1978, pp. 1-26. doi:10.1098/rspa.1978.0028

[3] T. B. Benjamin, "Bifurcation Phenomena in Steady Flows of a viscous Fluid II, Experiment”, Proceedings of the Royal Society A, Vol. 359, No. 1696, 1978, pp. 27-43. doi:10.1098/rspa.1978.0030

[4] T. Watanabe, H. Furukawa and I. Nakamura, "Nonlinear Development of Flow Patterns in an Annulus with decelerating Inner Cylinder”, Physics of Fluids, Vol. 14, No. 1, 2002, pp. 333-341. 
[5] H. Furukawa, T. Watanabe, Y. Toya and I. Nakamura, "Flow Pattern Exchange in the Taylor-Couette System with a Very Small Aspect Ratio”, Physical Review E, Vol. 65, No. 3, 2002, Article ID: 036306. doi:10.1103/PhysRevE.65.036306

[6] E. L. Koschmieder, "Bénard Cells and Taylor Vortices", Cambridge Univ. Press, Cambridge, 1993.

[7] J. Abshagen, O. Meincke, G. Pfister, K. A. Cliffe and T.
Mullin, "Transient Dynamics at the Onset of Taylor Vortices”, Journal of Fluid Mechanics, Vol. 476, 2003, pp. 335-343. doi:10.1017/S002211200200321X

[8] P. Manneville and O. Czarny, “Aspect-Ratio Dependence of Transition Taylor Vortices Close to Threshold”, Theoretical and Computational Fluid Dynamics, Vol. 23, No. 1, 2009, pp. 15-36. doi:10.1007/s00162-009-0093-x 